\title{
Different Techniques for Evaluation of Urologic Complication during Laparoscopic Gynecologic Surgery: A Review
}

\author{
1,2Tanitra Tantitamit, ${ }^{2}$ Kuan-Gen Huang
}

\section{ABSTRACT}

Aim: To summarize the previous studies in the literature regarding different strategies to detect urinary tract complication during intraoperative cystoscopy.

Background: The routine cystoscopy at the time of laparoscopic hysterectomy could be detected almost all bladder and ureteral injuries by visualizing ureteral jet after indigo carmine injection. Since, there was a shortage of Indigo carmine, various alternative methods have been suggested.

Review results: In this report, we conducted literature research in which 382 articles were identified. A total of 15 relevant articles were evaluated in full. We provide a review of the existing literature regarding the technique and effectiveness of each strategy in the evaluation of urologic complication during intraoperative cystoscopy. We also summarized and compared the properties, dosing, adverse effect, contraindication and cost among of each method.

Conclusion: There is not enough evidence to indicate the best strategy. Further studies are required. However, this information may guide the physicians to choose the most suitable method proper for their practices.

Clinical significance: Not enough data to determine which agent is superior to use at the time of cystoscopy. The surgeon should weight risks against benefits of each strategy. Not only the satisfactory but also the feasibility, safety, and costs should be considered.

Keywords: Cystoscopy, Gynecologic surgical procedure, Wound and injuries, Review, Ureter, Urinary bladder

How to cite this article: Tantitamit T, Huang KG. Different Techniques for Evaluation of Urologic Complication during Laparoscopic Gynecologic Surgery: A Review. J South Asian Feder Obst Gynae 2018;10(Suppl 1):335-339.

Source of support: Nil

Conflict of interest: None

${ }^{1}$ Assistant Professor, ${ }^{2}$ Associate Professor

${ }^{1}$ Department of Obstetrics and Gynecology, Faculty of Medicine, Srinakharinwirot University, Nakhonnayok, Thailand

2Department of Obstetrics and Gynecology, Chang Gung Memorial Hospital at Linkou and Chang Gung University College of Medicine, Kweishan, Taoyuan, Taiwan

Corresponding Author: Kuan-Gen Huang, Associate Professor, Department of Obstetrics and Gynecology, Chang Gung Memorial Hospital at Linkou and Chang Gung University College of Medicine, Kweishan, Taoyuan, Taiwan, Phone: +886-33281200, Ext 8253, e-mail: kghuang@ms57.hinet.net
Date of received: 5 March 2018

Date of acceptance: 10 November 2018

Date of publication: July 2019

\section{INTRODUCTION}

Urinary tract injury is the major complication following laparoscopic gynecologic surgery. Historically, laparoscopic hysterectomy has been a cause of greater frequency in urinary tract injuries when compared with abdominal or vaginal hysterectomy. This is due to the greater breadth of pathology and tendency to use electrosurgery that may lead to delayed thermal damage in laparoscopic surgery. ${ }^{1}$ Recently, the evidence showed the overall injury rate for laparoscopic hysterectomy was $0.73 \%$, which did not appear to differ from the abdominal approach. Bladder injury rate ranged $0.05-0.66 \%$ and the ureteral injury rate ranged $0.02-$ $0.4 \% .^{2}$ Delay diagnosis either bladder or ureteric injury is associated with serious morbidity. It can result in peritonitis, genitourinary tract fistula or renal damage. Intraoperative identification allows for immediate repair, decrease morbidity and improved outcome after surgery.

The routine cystoscopy at the time of laparoscopic hysterectomy is recommended by the American Association of Gynecologic Laparoscopists (AAGL) 2012 to detect most unsuspected the lower genital tract injuries. Only $25-50 \%$ of these injuries are recognized at the time of surgery if intraoperative cystoscopy is not performed. ${ }^{3}$ The result from a large retrospective study showed that there is up to a fivefold increase in intraoperative detected rates of urinary tract injury when cystoscopy is used after benign gynecologic surgery. ${ }^{4}$ Crude data from one systematic review found that the proportion of ureteric and bladder injuries detected intraoperatively without cystoscopy is approximately $18 \%$ and $79 \%$, respectively. These proportion increases to approximately $95 \%$ when routine cystoscopy is performed. Bladder injury is more likely to be detected up to 15 times compared with ureteric injuries. ${ }^{1}$ In addition, almost all bladder injuries and $80-90 \%$ of ureteral injuries are detected by intraoperative cystoscopy. The preferred method of recommendation according to AAGL is the visualizing ureteric ejection after injection of indigo-carmine. A normal cystoscopy with the efflux of dye cannot totally exclude ureteral injury, presumably secondary to either delayed thermal injuries or nonobtrusive injuries with delayed sequelae. ${ }^{3,4}$ 
There has been a shortage of indigo-carmine since $2014,{ }^{5}$ and now, it has become unavailable. Several alternative methods have been used instead methylene blue, sodium fluorescein, phenazopyridine, vitamin $\mathrm{B}$ or using other distension media by increasing dextrose concentration. The limited data showed different results of their effectiveness and satisfaction scores. The aim of this article is to summarize the previous studies in the literature regarding different strategies to detect urinary tract complication during intraoperative cystoscopy.

\section{REVIEW RESULTS}

We included studies that provide outcomes of the various methods in evaluation the urinary tract injury during intraoperative cystoscopy with information regarding properties, dosing, adverse effect, contraindication and cost of each method. A comprehensive, systemic literature search was undertaking on the PubMed database in the English language from January 1, 2000, to December 3, 2017. The search strategy used various combinations of the following; "urologic complication" "ureteric injury" "ureteral patency" "bladder injury" "intraoperative cystoscopy" "gynecologic laparoscopy".

Based on keywords, a total of 382 articles were identified. All abstracts were reviewed and 15 relevant articles were evaluated in full. The list of articles was supplemented by extensive cross-checking of reference lists of identified articles. Pertinent articles and reviews were used for the discussion.

\section{DISCUSSION}

We reviewed the literature and compared these available methods including the pros and cons list as presented in Table 1.

\section{Dextrose Distension Media}

As the different viscosity of dextrose solution and urine, using dextrose as the distension media should be seen the ureteric jet of urine clearly. One study compared the clarify in the visualization of ureteric jets between normal saline and 50\%Dextrose for use as distension media during cystoscopy. This study used the video clips demonstrated the use of both medium in the same patients as the assessment tool, and the results revealed that $50 \%$ dextrose is better to clarify the jets and preferred over normal saline. ${ }^{6}$ The concern is that the use of dextrose solution would cause glucosuria and increase the rate of urinary tract infection (UTI). A retrospective cohort study found that postoperative rates of UTI were higher in women underwent cystoscopy with 10\% Dextrose at the time of intraoperative cystoscopy than using normal saline. ${ }^{7}$ Flushing the bladder after finishing the cystoscopy could decrease the adherence of dextrose and uroepithelium. This may lead to a decrease in the postoperative rate of UTI. ${ }^{7}$ Further study is needed to support this concept. Another disadvantage of using dextrose media is it can cause sticky equipment following use.

\section{Dying Agent}

Methylene blue is a drug to treat methemoglobinemia from toxic substances and has been used off-label in a variety of procedure such as lymph node biopsy, endoscopic evaluation, urologic evaluation, and pulmonary nodule evaluation. ${ }^{8}$ It used in cystoscopy to color the urine blue, but the urine jets become lightly due to its metabolized. Joel et al. reported two patients in whom methylene blue was not visualized after received 1\% 10 $\mathrm{mL}$ intravenous. Both patients received subsequent doses and one patient received furosemide. The authors concluded that one-third of methylene blue was metabolized to leukomethylene which is colorless when excreted in the urine. ${ }^{9}$ The most common dose is $50 \mathrm{mg}$ or $1-2 \mathrm{mg} /$ $\mathrm{kg}{ }^{10}$ Verbeek et al. evaluated the use of methylene in 12 patients who underwent lower abdominal surgery. Methylene was injected intravenously at doses $0.25,0.5$, $1 \mathrm{mg} / \mathrm{kg}$ after exposure of the ureters. Near-infrared fluorescence imaging was performed for up to 60 minutes after injection. Both ureters in all patients could be clearly visualized within 10 minutes after administration. The author recommended using a low dose of 0.25 $\mathrm{mg} / \mathrm{kg}$. ${ }^{11}$ Methylene is a potent reversible inhibitor of monoamine oxidase inhibitor. This inhibition leads to serotonin toxicity when administered to patients on SSRI treatment. ${ }^{12}$ It is contraindicated in the patient taking the antidepressant, G6PD deficiency, renal impairment, and full-term pregnancy. ${ }^{10}$ Using this agent in cystoscopy is less common because of the unclear visualization and the safety concern as shown in Table 1 .

Fluorescein is the other options, but there are little experience and limited data. It routinely used for retinal angiography. The safety information of this agent based on the ophthalmologic literature. The incidence of an adverse reaction during retinal angiography reported only $1.28 \%$. The most common reactions are transient nausea $0.35-0.8 \%$, vomiting $0.15 \%$, urticarial $0.6 \%$ and hypoglycemia. ${ }^{13}$ A moderate reaction such as pyrexia or thrombophlebitis occurs less than $0.05 \%$. Only one in 11260 cases occurred bronchospasm and urticarial $(<0.01 \%){ }^{14}$ Doyle et al., recommend considering the use of $25 \mathrm{mg}$ of $10 \%$ sodium fluorescein intravenously to reduce the likelihood of adverse effects. In this study, 12 cases were injected with $10 \%$ sodium fluorescein in different doses during cystoscopy. It was more difficult to detect 


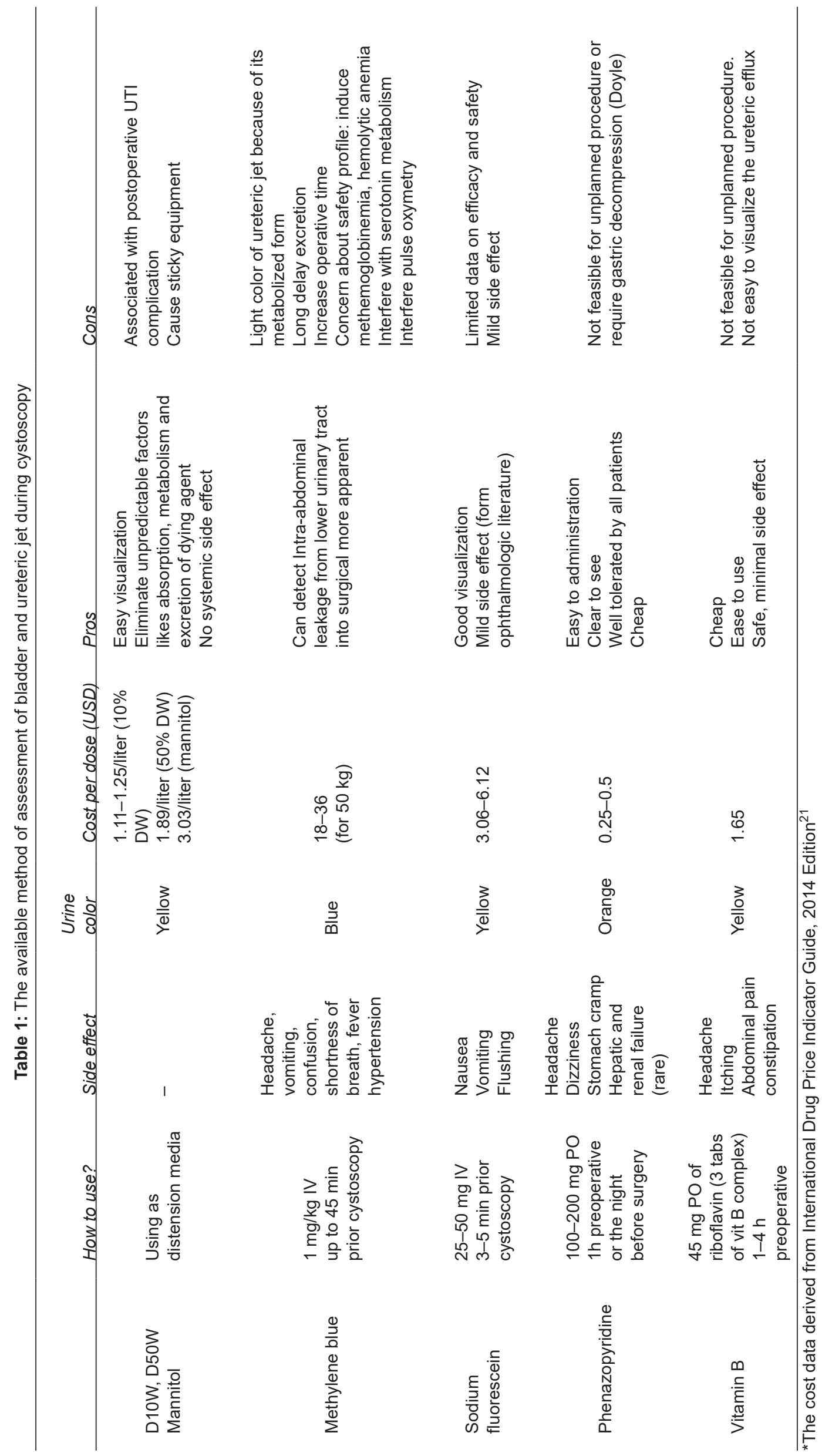


at $10 \mathrm{mg}$ dose, and one case experienced transient yellowing of sclera and palms after received $100 \mathrm{mg}$ dose. ${ }^{15}$ This agent should not be used in pregnancy of the patient who is allergic to the dye.

Phenazopyridine is a drug used for relief of burning pain from urgency and frequency caused by infection or trauma. The use and safety profile as a bladder analgesic has been well studied and showed no complications with use in a single short course. However, the physicians should avoid prescribing this drug in higher dose or longer duration than recommended especially in the patient with hepatic and renal impairment. Hui and colleagues reported the use of this drug to facilitate intraoperative cystoscopy in 32 women after pelvic reconstructive procedures. The patient received 100-200 mg of phenazopyridine one hour preoperatively. The efflux was visible in all cases. No missing ureteric injury was found. Ureteric patency could be confirmed rapidly by the evident orange-colored jets of urine. ${ }^{16}$ A larger study, randomized controlled trial of 104 women, compared the visualization of ureteral efflux between phenazopyridine group and no medication. The patient in the treatment group received $200 \mathrm{mg}$ of phenazopyridine on the morning of surgery. Time to visualize ureteral efflux did not differ between both groups ( 2 minutes 40 second in treatment and 2 minutes 53 seconds in control group). Nevertheless, the surgeon felt less frustrated and impatient in visualization in the phenazopyridine group. ${ }^{17}$ This drug should not be used in the patient with G6PD deficiency, renal or hepatic insufficiency.

Vitamin B is a water-soluble vitamin that colors the urine bright yellow. The advantage of this agent is cost and safety. When taken orally, the side effect is mild and rare even in high dose. From the first prospective study, 69 women were taken vitamin complex three tablets (45 mg of riboflavin), 1-4 hours before the operation. The bilateral yellow urine was visualized at the ureteric orifices in $72 \%$ of procedures. Others $(27.9 \%)$ had a unilateral jet or bilateral jet of clear or yellow urine. One case had a unilateral absence of ureteric jet and required indigo-carmine to confirmed the ureteric patency. Time to visualize both ureteric jets was 2.2 minutes, ranging from 0.5 to 10 minutes. ${ }^{18}$ The major limitation with the use of oral vitamin B is its light orange color. This may make the ureteric jets is not east to visualized.

\section{Comparing Different Modalities}

Two randomized control trials were conducted to compare different modalities in the evaluation of intraoperative cystoscopy in the post indigo carmine era. One study compared four groups: saline distention (control),
10\% dextrose distention, $200 \mathrm{mg}$ oral phenazopyridine, and intravenous sodium fluorescein. Sodium fluorescein resulted in more visible jets and higher surgeon satisfaction compared with others. Only sodium fluorescein and $10 \% \mathrm{D}$ resulted in significantly improved visibility and satisfaction when compared with the control group. UTI rates were similar among these interventions. Fluorescein resulted in very bright yellow tinge urine, which can be higher reflective and easier to detect when compared with phenazopyridine. $^{19}$

Another study compared the surgeon satisfaction with four methods: phenazopyridine, sodium fluorescein, mannitol, and NSS distension. Mannitol provided surgeons with the most satisfactory and superior visualization without affecting surgery or cystoscopy times. ${ }^{20}$ There is no difference in the rate of UTI among the groups. In this study, the patients with UTI were diagnosed by symptoms such as irritation and dysuria which might occur frequently due to Foley catheter retaining or manipulation during surgery. ${ }^{21}$ Therefore, we cannot exclude the possibility that using mannitol may increase UTI which is the concerned problem of this agent.

\section{CONCLUSION}

Not enough evidence to indicate the best strategy. Further studies are required. However, this information may guide the physicians to choose the most suitable method proper for their practices.

\section{CLINICAL SIGNIFICANCE}

Based on limited information from few studies which gave different results, there are not enough data to determine which agent is superior to use at the time of cystoscopy. It is not easy to design a satisfactory evaluation strategy to get a conclusion. The surgeon should weight risks against benefits of each strategy. Not only the satisfactory but also the feasibility, safety, and costs should be considered.

\section{REFERENCES}

1. Dassel MW, Adelman MR, et al. Recognition and management of urologic injuries with laparoscopic hysterectomy. Clin Obstet Gynecol 2015;58(4):805-811.

2. Adelman MR, Bardsley TR, et al. Urinary tract injuries in laparoscopic hysterectomy: a systematic review. J Minim Invasive Gynecol 2014;21(4):558-566.

3. AAGL Practice Report: Practice guidelines for intraoperative cystoscopy in laparoscopic hysterectomy. J Minim Invasive Gynecol 2012;19(4):407-411.

4. Tan-Kim J, Menefee SA, et al. Laparoscopic Hysterectomy and Urinary Tract Injury: Experience in a Health Maintenance Organization. J Minim Invasive Gynecol 2015;22(7):12781286. 
5. US Food and Drug Administration. FDA drug shortages. [Available from: https://www.accessdata.fda.gov/ scripts/drugshortages/default.cfm.

6. Narasimhulu DM, Prabakar C, et al. $50 \%$ dextrose versus normal saline as distension media during cystoscopy for assessment of ureteric patency. Eur J Obstet Gynecol Reprod Biol 2016;199:38-41.

7. Siff LN, Unger CA, et al. Assessing ureteral patency using $10 \%$ dextrose cystoscopy fluid: evaluation of urinary tract infection rates. Am J Obstet Gynecol 2016;215(1):74.e1-6.

8. Wheeler MCJ. Current Drug Shortages [updated November 2, 2017. Available from: https:/ /www.ashp.org/drug-shortages/current-shortages/Drug-Shortage-Detail.aspx?id=27.

9. Joel AB, Mueller MD, et al. Nonvisualization of intravenous methylene blue in patients with clinically normal renal function. Urology 2001;58(4):607.

10. Luketic L, Murji A. Options to Evaluate Ureter Patency at Cystoscopy in a World Without Indigo Carmine. J Minim Invasive Gynecol 2016;23(6):878-885.

11. Verbeek FP, van der Vorst JR, et al. Intraoperative near infrared fluorescence guided identification of the ureters using low dose methylene blue: a first in human experience. J Urol 2013;190(2):574-579.

12. Ramsay RR, Dunford C, et al. Methylene blue and serotonin toxicity: inhibition of monoamine oxidase A (MAO A) con- firms a theoretical prediction. Br J Pharmacol 2007;152(6):946951.

13. Jennings BJ, Mathews DE. Adverse reactions during retinal fluorescein angiography. Optometry 1994;65(7):465-471.

14. Beleña JM, Núñez M, et al. Adverse reactions due to fluorescein during retinal angiography. JSM Ophthalmol 2013;1:1004.

15. Doyle PJ, Lipetskaia L, et al. Sodium fluorescein use during intraoperative cystoscopy. Obstet Gynecol 2015;125(3):548-550.

16. Hui JYC, Harvey MA, et al. Confirmation of ureteric patency during cystoscopy using phenazopyridine $\mathrm{HCl}$ : a low-cost approach. JOGC 2009;31(9):845-849.

17. Propst K, Tunitsky-Bitton E, et al. Phenazopyridine for Evaluation of Ureteral Patency: A Randomized Controlled Trial. Obstet Gynecol 2016;128(2):348-355.

18. Fernando S, Dowling C, et al. The role of preoperative oral vitamin $B$ in the cystoscopic assessment of ureteric patency. Int Urogynecol J 2011;22(8):947-951.

19. Espaillat-Rijo L, Siff L, et al. Intraoperative Cystoscopic Evaluation of Ureteral Patency: A Randomized Controlled Trial. Obstet Gynecol 2016;128(6):1378-1383.

20. Grimes CL, Patankar S, et al. Evaluating ureteral patency in the post-indigo carmine era: a randomized controlled trial. Am J Obstet Gynecol 2017;217(5):601-610.

21. Frye JE. International drug price indicator guide. 2014th ed. Medford (MA):MSH; 2015. 